\title{
Ética militar: bastión para la Acción Integral ${ }^{1}$
}

\author{
https://doi.org/10.21830/9789585377141.03
}

\author{
Ivonn Alexandra Niño Medina ${ }^{2}$ \\ Escuela Superior de Guerra "General Rafael Reyes Prieto" \\ César Alberto Karan Benitez ${ }^{3}$ \\ Ejército Nacional de Colombia
}

\section{Resumen}

El presente capítulo tiene como objetivo caracterizar la ética militar como bastión de los soldados de Acción Integral. Con este propósito se realiza una investigación cualitativa-interpretativa de textos y experiencias que permitirá al lector comprender e interpretar la interrelación entre la ética militar y la Acción Integral. El estudio evidencia que un adecuado empleo de la Acción Integral disminuye las espirales de violencia, ya que al atender las causas que las generan, se aporta a la seguridad nacional mediante la reducción de las necesidades básicas insatisfechas, principalmente en regiones donde la ausencia de la institucionalidad ha generado espacios vacíos aprovechados por los actores que generan inestabilidad. Por lo tanto, se hace necesario que el Estado desarrolle operaciones constantes y sostenidas de Acción Integral anudadas a los demás asociados de la Acción Unificada.

Palabras clave: Acción Integral; Acción Unificada; ética; institucionalización del territorio; necesidades básicas.

1 Este capítulo presenta los resultados colaborativos de dos proyectos de investigación: (1) "Ética militar en entornos complejos de seguridad y defensa: lecturas y aportes desde la experiencia de las Fuerzas Militares de Colombia", del grupo de investigación Masa Crítica, de la Escuela Superior de Guerra "General Rafael Reyes Prieto", Colombia, categorizado en B por Minciencias y con código de registro COL0123247, y (2) "Mujeres de arma, seguridad y defensa nacional. Un análisis desde sus percepciones", del grupo de investigación en Ciencias Militares, de la Escuela Militar de Cadetes "General José María Córdova", Colombia, categorizado en B por Minciencias y con código de registro COL0082556. Los puntos de vista pertenecen a los autores y no reflejan necesariamente los de las instituciones participantes.

2 Magíster en Política Social de la Pontificia Universidad Javeriana. Magíster en Seguridad y Defensa Nacional de la Escuela Superior de Guerra. Profesional en Relaciones Internacionales y Estudios Políticos de la Universidad Militar Nueva Granada. OrCID: https://orcid.org/0000-0003-2049-3212 Contacto: ivonn.nino@esdegue.edu.co

3 Coronel del Ejército Nacional de Colombia. Profesional en Ciencias Militares. Especialista en Gerencia Pública de la Universidad Regional del Caribe. Especialista en Seguridad y Defensa Nacional de la Escuela Superior de Guerra. Diplomado en Derecho Internacional de los Derechos Humanos de la Universidad Autónoma de Bucaramanga. Diplomado en Docencia y Gestión Educativa del Centro de Educación Militar. ORCID: https://orcid.org/0000-0002-8849-0184 


\section{Introducción}

En el Estado colombiano se han presentado diversas situaciones sociales y culturales derivadas de la violencia ocasionada por el conflicto armado. Parte de ello, se fundamenta en que históricamente Colombia se ha visto inmersa en diversas polarizaciones y enfrentamientos que permean a la población, por lo cual se ha mantenido de manera constante y continua las espirales de violencia a partir de los espacios vacíos que genera la falta de institucionalidad. Lo anterior, en el entendido de que un conflicto, en palabras de Calduch (1993), es "una relación social por la que dos o más colectividades aspiran a satisfacer intereses o demandas incompatibles, utilizando sus desigualdades de poder para mantener actuaciones antagónicas o contrapuestas, recurriendo, en último extremo, a la violencia” (p. 91).

De esta manera, se crean factores que fomentan la inestabilidad de las regiones y contribuyen a la permanencia de las actividades ilícitas representadas en narcotráfico, microtráfico, explotación ilícita de yacimientos mineros, contrabando, entre otras, que dan cuenta de la constante presencia de los grupos armados organizados y grupos delincuenciales, que, en palabras de Álvarez y Zambrano (2019), representan la aparición de la dinámica de convergencia entre terrorismo, insurgencia y crimen organizado transnacional debido a los territorios sin gobierno efectivo dentro de estos Estados, a los cuales se les puede denominar "espacios vacíos"(p. 290).

Esta situación ha generado el desarrollo de estrategias lideradas por los diferentes gobiernos para intentar contrarrestar los factores que generan inestabilidad, dejando de lado la mitigación de las causas que los fomentan. Sin embargo, las Fuerzas Militares, a través de Acción Integral (ArN), han decidido emprender actividades de apoyo a la población para contribuir en la disminución de las necesidades básicas insatisfechas, en el entendido de que solo a partir de la combinación de estrategias se pueden lograr procesos de estabilización. No obstante, esta carrera no es posible si solo se realiza desde el sector defensa, por lo cual es necesario un trabajo coordinado y colaborativo entre todos los asociados de la acción unificada del Estado.

Por lo tanto, atendiendo a la importancia que la capacidad de Ain ha adquirido dentro de las Fuerzas Militares y, por ende, del Estado colombiano, 
vale la pena formular la siguiente pregunta: ¿De qué manera la ética militar debe ser transversal para el desarrollo de la Acción Integral? Para responderla, este capítulo resultado de investigación (1) establece el apoyo que Ain da al Estado colombiano, (2) identifica las actividades que desarrolla Ain y (3) define la importancia de la ética militar como variable transversal para el desarrollo de la AIN.

En la primera parte, el capítulo establece el marco teórico y conceptual que define las variables principales de la investigación, centrándose en los conceptos de acción integral, ética militar, necesidades básicas insatisfechas, espacios vacíos y acción unificada. En la segunda parte, presenta el método empleado para realizar la investigación y la forma como se analizó la información. En la tercera, se presentan otras posibilidades de interpretar los resultados y su contribución al tema en general, lo cual permite abrir puertas a nuevas investigaciones y preguntas. Finalmente, en la cuarta parte se desarrollan las conclusiones del estudio.

\section{Marco teórico y conceptual}

Con el fin de que el lector conozca las variables que forman parte del presente capítulo, a continuación se hace la relación de los conceptos transversales al estudio realizado. Se debe resaltar que a lo largo de la creación de Ain han existido diferentes definiciones para comprender su magnitud. Entre ellas se puede identificar la del general Mario Montoya, citado por Castillo y Niño (2020), quien la identifica como

el compendio de factores o elementos articulados y coordinados, para fortalecer las estructuras institucionales del Estado, en procura del bienestar de la colectividad, fundamentado en la legitimidad, la legalidad, el respeto a la dignidad humana, la protección y la difusión de los Derechos Humanos para todos los ciudadanos. (Montoya 2007, 1996; citado por Castillo \& Niño, 2020)

Si bien la capacidad de AIN genera resultados similares cuando es empleada por las diferentes Fuerzas Militares colombianas (Ejército Nacional, Fuerza Aérea y Armada Nacional), el concepto presenta enfoques distintivos 
en cada una de ellas. Para el Comando General de las Fuerzas Militares, la AIN se circunscribe a la potestad de "contribuir al esfuerzo de la Acción Unificada del Estado, de manera conjunta, coordinada, combinada e interinstitucional, con el fin de promover la prosperidad de la Nación" (Comando General de las Fuerzas Militares, s. f., s. p.). Por su parte, para la Fuerza Aérea, la Aın se realiza mediante la coordinación con instituciones públicas y del sector privado, para desarrollar una importante labor con la población, especialmente en las zonas más vulnerables a la acción delictiva de los grupos armados ilegales (Fuerza Aérea Colombiana, s. f.). Mientras que, para el Ejército Nacional, el concepto de Ain se ha incluido como término doctrinal, establecido por el Manual de campaña de Acción Integral, en el cual se indica que es

el conjunto de acciones militares que abarcan las operaciones de apoyo a la información militar, asuntos civiles, cooperación civil militar y asuntos públicos, las cuales permiten integrar las capacidades del Ejército a las de los asociados de la acción unificada, en apoyo a la intención del comandante. (Ejército Nacional de Colombia, 2019, p. 18)

Aunque las diferentes visiones apuntan hacia esfuerzos similares, en este capítulo se emplea la definición del Ejército Nacional, debido a que ha sido incluida en la doctrina y, por lo tanto, se hace más acertada su comprensión y empleo, en razón a que en esta se establecen "principios fundamentales que guían el trabajo de las unidades de una Fuerza en la acción coordinada hacia un objetivo común y puede incluir tácticas, técnicas, procedimientos y términos y símbolos" (Ejército Nacional de Colombia, 2017c, p. ix).

De la misma manera, se hace necesario indicar la relevancia de considerar dentro del marco conceptual la Acción Unificada, puesto que se basa en la necesidad de articular las capacidades de la fuerza militar con las instituciones públicas, privadas, organizaciones no gubernamentales y demás organizaciones a través de las cuales se puedan generar respuestas de atención a la comunidad para resolver las espirales violentas y cerrar los espacios vacíos. Esto, partiendo del interés que se lidera desde el nivel gubernamental, en donde se pretende emplear la "acción unificada, coordinada, interagencial, sostenida e integral del Estado, [ya que es] capaz de modificar substancialmente los contextos que favorecen el surgimiento, mantenimiento y reproducción de la criminalidad" 
(Ministerio de Defensa Nacional, 2019, p. 15) y de la participación que Arn realiza para aportar a los mencionados lineamientos.

Por ende, el concepto de Acción Unificada que se emplea en el capítulo es el que ha establecido el Ejército Nacional: "La sincronización, coordinación y/o integración de actividades de entidades gubernamentales y no gubernamentales con las operaciones militares para lograr unidad de esfuerzo" (Ejército Nacional de Colombia, 2019, p. 18).

Ahora bien, de acuerdo con los planteamientos de Moliner (2018), las democracias basadas en la libertad y la justicia, con todas sus imperfecciones, depositan en la organización militar, sometida al poder político, el uso legal de la fuerza (p. 3), por lo cual la ética es un factor fundamental e intrínseco en el desarrollo de las operaciones militares y del actuar de cada soldado.

Por lo tanto, para introducir el concepto de ética dentro de las actividades militares es necesario acudir a la filosofía enmarcada en lo que inició como una campaña institucional, conocida como "Comportamiento ético superior", entendido como "la fuerza interior que inspira a los integrantes del Ejército Nacional para lograr la victoria de manera irreprochable" (Ejército Nacional de Colombia, 2011, p. 12). El comportamiento ético superior es lo que hoy se conoce como una filosofía en las Fuerzas Militares, la cual configura una cultura representativa para la formación del soldado, pero también como base para el desarrollo de cada una de las actividades en las que se desenvuelva la fuerza militar. La ética es en definitiva un saber para fundar y orientar racionalmente la acción humana hacia lo bueno, lo válido o lo correcto (Moliner, 2018, p. 6).

A su vez, la ética profesional militar "se fundamenta en principios, valores, virtudes y competencias, donde cada uno debe ser capaz de hacer propio el legado moral de la cultura castrense, donde se siguen las pautas de actuación establecidas con vocación de servicio" (Comando General de las Fuerzas Militares, 2016, p. 11). La ética es trascendente en la vida del ser humano y más en quienes tienen el oficio del uso legítimo de las armas del Estado, en la incansable tarea de lograr la paz (Comando General de las Fuerzas Militares, 2016, p. 11). De ahí parte la condición y voluntad del soldado y los valores humanos que le permiten conocer e identificar las áreas donde las actividades 
de Ain realmente aporten a la reducción de los factores que generan inestabilidad y contribuyan a disminuir los espacios vacíos que fomentan la ilegalidad.

De esta manera, en este capítulo se entiende por ética militar "la construcción del tejido cultural que reviste a las Fuerzas Militares del honor y la legitimidad que se requieren para mantener la confianza de los conciudadanos en el ejercicio de sus funciones" (Comando General de las Fuerzas Militares, 2016, p. 26).

Para comprender la magnitud del aporte que hace Ain se acude al concepto de necesidades básicas, que la Unesco define como las "condiciones mínimas indispensables para tener un nivel de vida decente: comida adecuada, techo, ropa y servicios comunitarios. Incluyen también necesidades relacionadas con los Derechos Humanos, la participación en las decisiones colectivas y un trabajo remunerado" (Unesco, s. f.). En palabras de Hayek (citado por Álvarez, 2003), este concepto aplica a la conjunción de diferentes formas de reproducción:

Por ejemplo, en alimentación, se trata de la cobertura de una canasta básica de alimentos para acceder a las calorías y las proteínas necesarias al límite de la indigencia. A partir de los inicios de los noventa, las distintas instituciones del Sistema de Naciones Unidas promueven niveles básicos. En salud, la Organización Mundial de la Salud habla de paquetes básicos: provisión de atención primaria de la salud, cuidado de la embarazada y protección de niños en situación de riesgo. En saneamiento se trata de provisión de agua potable y de saneamiento básico. En educación, la Conferencia de Jomtien promueve las necesidades educativas básicas (Neba), lo que se traduce en el lema "educación para todos" solo a nivel de educación básica o primaria. (p. 250)

En referencia a la institucionalización, Díez et al. (2015) consideran que es "un proceso complejo y difícil de implementar que requiere la puesta en marcha de iniciativas por parte de sus gestores" (p. 17). De ahí que la apuesta de la institucionalización del territorio sea entendida como "la presencia activa y sostenida de las instituciones del Estado y con ello el acatamiento del ordenamiento jurídico a partir de la Acción Unificada del Estado" (Ministerio de Defensa, 2019, p. 63). En palabras de Álvarez (2017), es "el establecimiento de las capacidades institucionales necesarias para corregir las condiciones que favorecen el control ilegal del territorio, logrando así el imperio de la Constitución 
y la ley. Es decir, lograr que el Estado obtenga el monopolio de la seguridad, la justicia y la tributación” (p. 382).

\section{Métodos}

Pérez (2004; citado por Martínez, 2013) afirma:

El conocimiento de la realidad consiste en acercarse a ella, develarla y conocerla, con el fin de mejorarla, pues la realidad es algo que nos viene dado, lo que existe, el ámbito en el que se desarrolla la vida del hombre y todo aquello con lo que se relaciona. Implica el saber dónde se está, a dónde se quiere ir y cómo hacerlo. (p. 1)

De acuerdo con esta perspectiva, esta investigación emplea como método la hermenéutica textual, que, de acuerdo con González (2013), es "la teoría de las operaciones de comprensión en su relación con la interpretación de textos, en la que la palabra hermenéutica no significa otra cosa que la experiencia metódica de la interpretación" (p. 59). Para Cárcamo (2005), un elemento esencial para la comprensión en el proceso de interpretación es "la focalización precisa respecto a aquello que se desea interpretar" (p. 208).

En concordancia, el diseño de la investigación es cualitativo-interpretativo, desde el enfoque de la etnometodología, la cual, según Ghiso (1996), estudia "los fenómenos sociales incorporados a nuestros discursos y nuestras acciones a través del análisis de las actividades humanas [...] para centrarse en el estudio de los métodos o estrategias empleadas para construir, dar sentido y significado a sus prácticas sociales cotidianas (p. 10)”. Específicamente, se realizó una revisión sistemática de literatura, que, de acuerdo con Manterola et al. (2011), consiste en "una revisión de aspectos cuantitativos y cualitativos de estudios primarios, con el objetivo de resumir la información existente respecto de un tema en particular" (p. 10).

\section{Discusión}

Las dinámicas cambiantes de la sociedad colombiana, representadas en variables que se derivan de la inmersión de todo un país en un conflicto 
armado durante más de 60 años, han significado una lucha constante por parte del Estado para intentar disminuir los factores que han generado inestabilidad en los sectores político, económico y social. Según Rojas (2019), en la actualidad las dinámicas de violencia perpetradas por actores ilegales han incidido en la creación y permanencia de vulnerabilidades para las poblaciones colombianas, a causa de una larga trayectoria de violencia heredada del conflicto armado interno (p. 15), el cual ha dejado 8’376.463 víctimas (Registro Único de Víctimas [Ruv], 2017).

La dinámica del conflicto ha sido en gran parte debido a lo que Orjuela (2000) argumenta como “incapacidad histórica para integrar el territorio nacional [...] [y a que] el Estado colombiano no integra ni cohesiona la población ni el territorio y ha reducido su capacidad para mediar y canalizar los conflictos y tensiones sociales" (p. 104). En este sentido, Zartman (1995) describe que la "ausencia institucional del Estado permite que otras organizaciones tengan prioridad en la determinación de las reglas de la vida cotidiana” (p. 257), razones que conllevan una continua permanencia de las espirales violentas.

Sumado a ello, se han librado diferentes estrategias lideradas por el alto nivel del gobierno, en donde la constante ha sido combatir el narcotráfico, el secuestro, la extorsión e, incluso, intentar desintegrar los grupos armados organizados y los grupos delincuenciales, bien sea a partir del despliegue de operaciones militares o a través de una serie de intentos de definir procesos de paz, entre otros. Sin embargo, al detenerse en estos aspectos, se observa que estas estrategias han tenido como constante combatir las consecuencias de la ausencia institucional en los territorios, la carencia para dar respuesta a las necesidades de las comunidades, la falta de oportunidades y el poco desarrollo que se vive en las regiones que cuentan con mayores variables delincuenciales. Así, las estrategias que se han gestado para contrarrestar las consecuencias violentas le han dejado un mínimo esfuerzo para enfrentar las causas que las generan.

Según Álvarez \& Zambrano (2019), en estas regiones es donde se desarrollan generalmente los grupos delincuenciales debido a que han sido áreas descuidadas por el Estado, como los espacios vacíos rurales o urbanos (comunas, favelas, cinturones de miseria), en comunidades que a menudo 
sufren una falta de oportunidades, a las cuales responden creando sus propias fuentes de trabajo y de seguridad (p. 281). Tal situación no es ajena para el gobierno nacional, en la medida en que el emprendimiento y la equidad se indica en la Política de Defensa y Seguridad para la Legalidad:

La insuficiente respuesta del Estado para ocupar institucionalmente los espacios geográficos en los que delinquían estructuras desmovilizadas de las FARC, facilitó la incursión y el mantenimiento de diversos aparatos armados, configurándose hoy en día como uno de los principales obstáculos para lograr el imperio de la ley, el orden democrático y el ejercicio de los derechos de los ciudadanos. (Ministerio de Defensa Nacional, 2019, p. 28)

En este escenario, las Fuerzas Militares han desarrollado capacidades centradas en aportar a la disminución de la violencia y de las necesidades básicas insatisfechas de las comunidades con mayores índices de vulnerabilidad, en razón a que "ningún conflicto tiene una solución puramente militar y el éxito de la misión requiere el apoyo de las autoridades y de la población civil” (Ejército Nacional de Colombia, 2019, pp. 1-3). Así, con años de experiencia a partir de un trabajo colaborativo entre la institución castrense y la comunidad, surgen las relaciones civiles y militares, las cuales con el paso del tiempo se han fortalecido en dimensión y misionalidad, para convertirse en lo que hoy se conoce como Acción Integral.

La Acción Integral nació como el profundo anhelo de los hombres del Ejército Nacional, que buscaban servir a través de la vocación, la integración y el desarrollo a la población civil. Para tal fin, crearon un modelo que garantiza el trabajo social institucional en los lugares más apartados del territorio nacional, llevando un sinnúmero de actividades sociales a poblaciones específicas. (Comando General de las Fuerzas Militares, 2020a)

Las tácticas y los procedimientos de esta capacidad se materializan gracias a la transformación que tiene el Ejército Nacional a partir de la generación y actualización de los manuales con la doctrina Damasco. En tal sentido, Rojas (2019) afirma que "bajo los parámetros de Damasco, Acción Integral fortalece su papel en la acción decisiva, siendo una capacidad dotada de experiencia nacional en el acercamiento de la comunidad, así como su acompañamiento antes, durante y después de las operaciones militares" (p. 17). 
La doctrina de Ain se contempla en los manuales de campaña $M C E$ 3-53.0 Acción Integral y MCE 3-53 Operaciones de apoyo a la información militar. En ellos, se identifican cuatro disciplinas a partir de las cuales se desarrollan las actividades que interactúan con la comunidad, los asociados de la Acción Unificada, los enemigos y adversarios del Estado. Estas disciplinas son: asuntos civiles, operaciones de apoyo a la información militar, asuntos públicos y operaciones de apoyo a la información militar. Un acierto por demás para el Estado colombiano, ya que en palabras de Montoya (2007), "el concepto de Acción Integral es una estrategia adecuadamente integrada a la totalidad de acciones militares para ganar la guerra" (p. 19).

Debido a la relevancia que Ain ha adquirido a lo largo de su construcción y fundamentación, es necesario que el personal que se especializa en esta capacidad sea un administrador de la profesión del Ejército, quien debe garantizar que la profesión mantenga las seis características esenciales ahora y en el futuro, a fin de que se esfuerce continuamente por la excelencia en el desempeño del deber y administre de manera eficiente, efectiva y ética los recursos, la propiedad, los sistemas y las instalaciones del Ejército (Ejército Nacional de Colombia, 2017c, p. 17).

Otro de los factores que ha demostrado un avance significativo para Ain está representado en la capacidad de respuesta para atender las situaciones de emergencia nacional, bien sean estas ocasionadas por la naturaleza o por el ser humano. Estas actividades se describen en el Manual de apoyo de la defensa a la autoridad civil, conocido como ADAC y en el cual se delega la responsabilidad a Ain para que

lidere el direccionamiento del trabajo interinstitucional y de esta manera coordine con los diferentes ministerios o las instituciones que ejecuten funciones a nivel nacional para apoyar a las autoridades civiles, así como a los ingenieros militares en la gestión del riesgo de desastres. (Ejército Nacional, 2017b, p. 35)

Una tarea que resulta de gran importancia para apoyar a las comunidades a disminuir sus necesidades en situaciones coyunturales críticas. Parte de su importancia recae en que el personal de Ain centraliza e incluye de manera transversal en sus actividades los siete propósitos del ADAC: 
Salvar vidas; restaurar los servicios esenciales; mantener o restaurar la ley y el orden; proteger la infraestructura y propiedad (pública y privada); apoyar el mantenimiento o restauración del gobierno local; configurar el ambiente operacional para el éxito interagencial, y apoyar con la recuperación social del territorio. (Ejército Nacional de Colombia, 2017b, p. 16)

Así, es necesaria una adecuada articulación entre los demás asociados de la Acción Unificada, la empresa privada y la comunidad para conformar frentes sólidos que permitan avanzar en procesos de estabilización (Ejército Nacional de Colombia, 2019, p. 25), en la cual, según Fernández-Santillán (2018), se "requiere de un diálogo entre las personas comunes y corrientes y los administradores públicos [sobre] muchos tópicos y problemas que pueden ser resueltos mediante la participación convergente de estos actores en el espacio público" (p. 185).

De ahí que la armonización y el diálogo permitan identificar las necesidades reales de las comunidades, de tal manera que la articulación de estos actores facilite poner en marcha la implementación de planes y programas que, de un lado, contribuyan a minimizar las causas no atendidas que derivan en una espiral sin fin de violencia y, de otro, faciliten desde un trabajo colaborativo la atención de situaciones de emergencia. En palabras de Montero et al. (2008), esto contribuirá a que se logre direccionar soluciones a las necesidades reales de las personas, haciendo que la confianza social esté asociada a indicadores de éxito y de bienestar (p. 15).

\section{Actividades desarrolladas por Acción Integral}

Para que Ain pueda desarrollar un trabajo que aporte a las comunidades, es necesario el desarrollo metódico y organizado de sus actividades. En ese sentido,

esta especialidad funciona a través de un engranaje que le permite a la institución asegurar un trabajo ético y superior, dentro del que encontramos: al Departamento de Acción Integral y Desarrollo (CEDE9), que encamina su misión al diseño de planes, políticas y lineamientos; la Escuela de Misiones Internacionales y Acción Integral (EsMAI), la cual tiene como misión capacitar, entrenar y especializar a quienes cuenten con el perfil para trabajar en Acción Integral; y por último, al Comando de Apoyo de Acción Integral y 
Desarrollo (CAAID), que en coordinación con entidades gubernamentales y no gubernamentales, integra las capacidades de las Fuerzas Militares con los asociados de la Acción Unificada mediante la sincronización, coordinación e integración, generando las condiciones para promover la recuperación social en las comunidades más vulnerables. (Comando General de las Fuerzas Militares, 2020b, s. p.)

Las actividades se planean desde el Departamento de Acción Integral y Desarrollo, el cual tienen como lineamientos

planear, fijar y mantener las relaciones, generar lazos de trabajo mancomunado y establecer canales de comunicación entre la Fuerza y las autoridades políticas y judiciales, así como con representantes de la sociedad, comunidades étnicas y campesinas con el fin de facilitar la interacción con ellos; promover acercamientos entre la Fuerza, los asociados de la oferta estatal (Acción Unificada) y la comunidad, que apoyan el cumplimiento de la misión en todas las tareas de la acción decisiva, y orientar los lineamientos para fortalecer la imagen institucional con actividades de información pública y de encuentro con la comunidad dirigida tanto a públicos externos como internos con interés en el sector defensa. (Ejército Nacional de Colombia, s. f., s. p.)

Por su parte, el Comando de Apoyo de Acción Integral y Desarrollo es la unidad que se encarga de operacionalizar las actividades que realiza la capacidad, demostrando en su misión la conducción

de operaciones de Apoyo de la Defensa a la Autoridad Civil (ADAC) y estabilidad, en apoyo a las unidades del Ejército Nacional y en coordinación con las instituciones de Estado y de gobierno, con el fin de generar las condiciones para promover la recuperación social del territorio, en camino a la consolidación. (CAAID,s. f., s. p.)

En este sentido,

a través de herramientas y actividades como: circos institucionales, grupos musicales, Vehículos Modulares de Acción Integral (VemaI), emisoras, perifoneo táctico, puestos de control, máquinas duplicadoras, volantes, jornadas humanitarias, ambientales, entre otras; se ha convertido en parte importante de la transformación de la Fuerza y que consolida a los hombres de Acción Integral como soldados preparados para aportar al desarrollo del país. (Comando General de las Fuerzas Militares, 2020a, s. p.) 
La organización y puesta en marcha de la Ain representa un trabajo colaborativo, que según Serra (2008) "rechaza la idea de que el gobierno y la sociedad fuesen enemigos; desecha la mentalidad de que lo que uno gana el otro lo pierde, y viceversa, y resalta la idea de que ambos sectores pueden ser aliados" (p. 182), dado que para su desarrollo es necesario un constante diálogo con las comunidades, en el cual el valor público se centra en el interés por disminuir las vulnerabilidades sociales.

Existen amplios ejemplos de los resultados de Ain en apoyo al desarrollo de las operaciones militares, ya que "no basta con el tradicional despliegue de los recursos de la fuerza legítima del Estado, si no se consigue una efectiva acción articulada, coordinada, interagencial y unificada de las instituciones y la ciudadanía" (Ministerio de Defensa Nacional, 2019, p. 6).

En palabras de Karan y Niño (2018), Ain tiene:

una enorme responsabilidad asumida por todos los integrantes de la institución castrense en cumplimiento de la misión constitucional que ha permitido el desarrollo de capacidades que aportan paralelamente a las operaciones militares, las cuales, sin duda, contribuyen a minimizar la afectación a la seguridad del país. (p. 20).

Esto ha implicado que emerjan programas liderados por AIn a través de los cuales se aporte a la institucionalización del territorio, como es el caso de Fe en Colombia, el cual constituye "una respuesta innovadora para apoyar la disminución de las vulnerabilidades de las comunidades, a partir de un diagnóstico desde el territorio [...] minando así la posibilidad de que la criminalidad encuentre en la población formas de accionar sus actividades ilícitas" (Rojas, 2019, p. 17).

Por lo tanto, el desarrollo de las actividades de Ain requiere del aporte significativo de los asociados de la Acción Unificada, pues, según López (2017), es necesario que "se consolide la Acción Unificada del Estado aprovechando las capacidades militares mediante una verdadera presencia que permanezca en el tiempo, y que no solo se limite al aspecto de seguridad, sino que genere infraestructura, salud, educación y fuentes de ingreso que sustituyan las economías ilegales" (p. 83), para que de esta manera se permita al Estado colombiano generar la institucionalidad. 
Existen innumerables ejemplos de la contribución de Ain al desarrollo de las comunidades, en las cuales se realizan actividades que van desde la lúdica y la recreación, hasta la puesta en marcha de obras y proyectos que mejoran considerablemente la calidad de vida de las comunidades. A manera de ejemplo, se puede observar cómo

en el departamento del Caquetá, la Sexta División del Ejército Nacional a través de una alianza entre su programa Fe en Colombia con la Cámara de Comercio de Florencia, realizó una caracterización a 14 artesanos de la región e inició el diseńo de un catálogo virtual para visibilizar estos emprendimientos a gran escala. Este proceso tiene como finalidad tener un sistema de información que permita identificar qué materias primas transforman estas personas para convertirlas en bienes, a cuántas personas de su núcleo vinculan en las actividades que ejercen, así como el número de empleos que generan y el método de comercialización que han adoptado. Con el objetivo de mejorar las ventas de estos pequeños comerciantes, durante la etapa de aislamiento obligatorio preventivo, el Ejército Nacional a través del equipo de profesionales de su programa Fe en Colombia, se encuentra realizando un catálogo virtual con el que estos artesanos, divididos en tres grupos: bisutería, gastronomía y artículos para el hogar, podrán promocionar sus productos en redes sociales, dando a conocer información sobre sus historias de vida y generalidades de las técnicas empleadas. (Comando General de las Fuerzas Militares, 2020b, s. p.)

En el mismo sentido, a través del programa Fe en Colombia y en coordinación con la Agencia de Desarrollo Rural se realizó en Otanche, Boyacá, la Feria de Desarrollo Rural, la cual tuvo como objetivo generar oportunidades reales en la construcción del campo con futuro, que promueve el emprendimiento rural y así contribuir a la transformación del agro colombiano. Los grandes beneficiados de esta iniciativa fueron los pequeños empresarios locales, que tuvieron la oportunidad de comercializar sus productos con propios y turistas (Comando General de las Fuerzas Militares, 2019).

De esta manera, Arn se constituye como un eje articulador de la institución castrense que aporta a la Acción Unificada para fomentar escenarios que vislumbren opciones de interés, de acuerdo con las necesidades de las comunidades y proyectando de manera sostenida y sostenible estrategias que permitan minimizar las espirales violentas y así contribuir a la mejora de la seguridad nacional. Evidentemente, solo a través de un direccionamiento gubernamental 
que impulse a los asociados de la Acción Unificada a emprender actividades conjuntas, coordinadas y combinadas se logrará cerrar los espacios vacíos y fomentar la institucionalización del territorio.

\section{Ética militar en las actividades de Acción Integral}

Para lograr los resultados que se esperan con Ain en beneficio de las comunidades se necesita que el personal se encuentre debidamente preparado, de forma que puedan identificar las áreas de oportunidad y, de esta manera, disminuir las necesidades básicas insatisfechas y forjar los cierres de los espacios vacíos que se derivan en criminalidad mediante la apertura a la institucionalización del territorio. En este sentido, mediante la aplicación de la Ain se configura una articulación institucional adecuada, "ya que las capacidades de las Fuerzas se pueden aprovechar para fortalecer su relación con las comunidades desde una óptica de atención y prevención" (Ministerio de Defensa, 2019, p. 66).

Los hombres y las mujeres de Ain deben contar con competencias éticas que les permitan conocerse a sí mismos como profesionales militares, de tal modo que la autoevaluación los acredite con una adecuada capacitación para saber qué tienen que hacer, cómo lo deben hacer y en dónde lo deben hacer, a fin de que su trabajo cuente con una buena orientación y los beneficiarios queden completamente satisfechos con los resultados.

En consecuencia, la ética debe ser el núcleo de las actividades desarrolladas por los soldados de Arn, evitando que los prejuicios, las creencias y las emociones personales participen en la toma de decisiones sobre las áreas o comunidades que requieran el despliegue de capacidades tanto de la fuerza militar como de los demás asociados de la Acción Unificada. En palabras de Moliner (2018),

para evitar el relativismo cultural de los valores morales y reconociendo la complejidad de la realidad y que en nuestro planeta existen diversas civilizaciones y culturas, se defiende desde un punto de vista axiológico que existe una ética fundada en los valores y principios esenciales recogidos en la Declaración Universal de Derechos Humanos de 1948, convertida en código moral de aspiración moral. (p. 9) 
Con esta premisa, la ética fundamenta el deber del soldado de Ain, pues para que aporte a la población su actividad deberá estar alineada con los preceptos y las normas de respeto al Derecho Internacional de los Derechos Humanos y al Derecho Internacional Humanitario. Así mismo, siempre deberá guardar moderación por las actuaciones transparentes que preserven la confianza, el apoyo público, el buen nombre y la imagen institucional (Ejército Nacional de Colombia, 2019, p. 27).

De tal manera, para que los soldados de Ain puedan hacer aportes significativos, deben generar dinámicas que permitan comprender que la victoria militar no solo implica un enfrentamiento armado. Por esto fue necesario contribuir como institución a mitigar el impacto social derivado de la falta de cobertura de las necesidades básicas insatisfechas de poblaciones asentadas en regiones apartadas, sin suplantar la misionalidad de las demás instituciones del Estado. (p. 21)

Por lo anterior, la ética militar deberá reflejarse desde un enfoque educacional en el conocimiento de la oferta estatal a fin de facilitar "la implementación de programas de cooperación interorganizacional que permitan consolidar ganancias" (Ejército Nacional de Colombia, 2019, p. 84). Al respecto, Castellanos (2015) asegura que es necesario que el profesional militar desarrolle y emplee una destreza ética especial y singular que contenga como esencia las características de los valores que requiere nuestra sociedad (p. 13).

Además, los cambios en la dinámica operacional obligan a los soldados de Ain a ser activos y veloces en la toma de decisiones, lo cual debe entenderse y comprenderse dentro del ambiente de su área de operaciones y materializada a partir de un adecuado proceso de operaciones (planear, preparar, ejecutar y evaluar), ya que en cada uno de estos pasos se debe contar con el sentido ético en el actuar de la operación.

Si bien las unidades de las Fuerzas Militares desplegadas en todo el país, así como las unidades de Carabineros, son clave en la solución de problemas inmediatos de la población y decisivas para impactar positivamente en el mundo rural, construir cultura de legalidad y denegar el territorio a la delincuencia (Ministerio de Defensa, 2019, p. 66), esto no implica que se genere una extralimitación de la misionalidad de la institución, por lo que, para entender su funcionalidad, es necesario que se vincule el deber ético de cumplir con dili- 
gencia, eficiencia e imparcialidad las responsabilidades que les sean encomendadas (Comando General de las Fuerzas Militares, 2016, p. 24), acudiendo en los casos pertinentes a la armonización de las capacidades que posibiliten un mayor dinamismo para alcanzar la institucionalización del territorio.

Por ello, la doctrina de Ain busca estandarizar las tácticas y los procedimientos que se emplean, ya que de esta manera se establecen y fomentan los rasgos de carácter deseables en líderes y soldados, a quienes debe exigírseles la iniciativa, la creatividad, la capacidad de adaptación y la acción ética (Ejército Nacional de Colombia, 2017c, p. 30). Además, se debe tener en cuenta que armonizar las capacidades entre la institución y los demás asociados de la Acción Unificada para dar respuesta a las necesidades básicas insatisfechas permite que el soldado de Ain acceda a recursos materiales o humanos, por lo cual es fundamental que cuente con una eficiente formación ética profesional militar antes de ser desplegado en un teatro de operaciones, pues de esa manera se procura evitar que realice acciones antiéticas (Castellanos, 2015, p. 4). En virtud de lo anterior, también se hace hincapié en la importancia de evitar la creación de falsas expectativas en la sociedad, de manera que la ética militar se debe contemplar como factor fundamental para disminuir la posibilidad de que los soldados intenten acercarse a una comunidad con promesas que no se pueden cumplir.

\section{Conclusión}

La naturaleza de la violencia en Colombia y los espacios vacíos generados por la ausencia de la institucionalidad en diversas regiones han configurado escenarios de oportunidad para que el accionar criminal se desarrolle y tenga continuidad en representación de los grupos armados y de la delincuencia organizada, de tal manera que se convierten en el enfoque de atención del sector gubernamental. Como consecuencia, los planes de gobierno se han concentrado en combatir los factores que generan inestabilidad, pero han dejado de lado las causas que los originan.

Sin embargo, la comprensión que tiene la institución militar sobre la configuración del área de operaciones le ha enseñado que es necesario aportar a 
la disminución de las necesidades básicas insatisfechas mediante las actividades de Ain, con las cuales se logra fomentar la articulación con los demás asociados de la Acción Unificada a fin de fomentar la institucionalización del territorio.

De este modo, al mantener de manera constante relaciones con el sector civil — representado por las instituciones del Estado, las entidades privadas, las organizaciones no gubernamentales y la comunidad en general—, los soldados de Ain deben contar con la investidura de la ética como bastión para el desarrollo de sus actividades. Lo anterior, en razón a que el valor ético construye eslabones de comportamiento que se soportan en lo que se hace bien y para hacer el bien, pero sobre todo por el respeto a los Derechos Humanos.

Así, las consideraciones que se hacen en este capítulo constituyen un aporte significativo porque, por una parte, permiten comprender el fundamento de Ain y su participación activa como capacidad de la institución militar para contribuir a la disminución de los factores que generan inestabilidad. Por otra parte, este trabajo evidencia que el aporte de Ain a las comunidades consiste en proponer soluciones que fomenten la atención a las necesidades básicas insatisfechas, razón por la cual la ética, en cuanto condición natural de Arn, debe armonizar sus capacidades para que no se traspasen los límites de la misionalidad militar ni se generen falsas expectativas en la comunidad.

\section{Referencias}

Álvarez, C. (2017). Ocupación de espacios vacíos: Una condición sine qua non de la seguridad multidimensional en Colombia. En C. E. Álvarez Calderón (ed.), Desafíos y nuevos escenarios de la seguridad multidimensional en el contexto nacional, regional y hemisférico en el decenio 2015-2025 (pp. 307-386). Escuela Superior de Guerra. https://esdeguelibros.edu. co/index.php/editorial/catalog/view/27/23/313

Álvarez, C., \& Zambrano, J. C. (2019). Globalización desviada: Plataforma de convergencia criminal. En C. E. Álvarez Calderón (ed.), Desafios y nuevos escenarios de la seguridad multidimensional en el contexto nacional, regional y hemisférico en el decenio 2015-2025 (pp. 250-304). Escuela Superior de Guerra. https://esdeguelibros.edu.co/index.php/editorial/ catalog/view/27/23/313

Álvarez Leguizamón, S. (2003). Los discursos minimistas sobre las necesidades básicas y los umbrales de ciudadanía como reproductores de la pobreza. En Trabajo y producción de la pobreza en Latinoamérica y el Caribe, estructuras, discursos y actores. Consejo Latinoamericano de Ciencias Sociales. 
Calduch, R. (1992). Las relaciones internacionales. https://www.ucm.es/data/cont/media/www/ pag-55159/lib1cap4.pdf

Cárcamo V., \& Héctor. (2005). Hermenéutica y análisis cualitativo. Cinta de Moebio. Revista de Epistemologia de las Ciencias Sociales, (23), 204-216. http://www.facso.uchile.cl/publicaciones/moebio/23/carcamo.htm

Castellanos, C. (2015). La influencia de la ética profesional militar en el teatro de operaciones. Escuela Superior de Guerra de las Fuerzas Armadas de Argentina. http://cefadigital.edu. ar/bitstream/1847939/499/1/TFI\%2004-2015\%20CASTELLANOS.pdf

Castillo, A., \& Niño, C. (2020). La doctrina de Acción Integral como política de seguridad en el posconflicto armado en Colombia. En Perspectivas y prospectivas de la seguridad en Colombia (pp. 121-148). Universidad Santo Tomás. https://www.researchgate.net/publication/322505438_La_Doctrina_de_Accion_Integral_como_politica_de_seguridad_en_ el_posconflicto_armado_en_Colombia

Comando de Apoyo de Acción Integral y Desarrollo [CAAID]. (s. f.) Misión. https://www.caaid. mil.co/comando_apoyo_accion_integral_desarrollo/conozcanos/mision

Comando General de las Fuerzas Militares. (2016). Manual de generalidades éticas 1-5 (1. a ed.). Imprenta y Publicaciones de las Fuerzas Militares. https://www.mindefensa.gov.co/irj/ go/km/docs/Mindefensa/Documentos/descargas/Sobre_el_Ministerio/Control_Interno/ documentos/manual_generalidades_eticas_FFMM.pdf

Comando General de las Fuerzas Militares. (2019, diciembre 17). Fe en Colombia y el Ejército se une a la feria construyendo desarrollo rural. https://www.cgfm.mil.co/es/blog/fe-en-colombia-y-el-ejercito-se-une-la-feria-construyendo-desarrollo-rural

Comando General de las Fuerzas Militares. (2020a, agosto 9). El Ejército Nacional celebra el 4. ${ }^{\circ}$ aniversario de la especialidad de Acción Integral. https://www.cgfm.mil.co/es/blog/ el-ejercito-nacional-celebra-el-4o-aniversario-de-la-especialidad-de-accion-integral

Comando General de las Fuerzas Militares. (2020b, julio 19). Ejército Nacional apoya a artesanos del departamento del Caquetá. https://www.cgfm.mil.co/es/blog/ejercito-nacional-apoya-artesanos-del-departamento-del-caqueta

Comando General de las Fuerzas Militares. (s. f.). Misión Departamento Conjunto de Acción Integral y Desarrollo. https://www.cgfm.mil.co/es/mision-departamento-conjunto-de-accion-integral-y-desarrollo

Díez, E., Díez, F., \& Vásquez, A. (2015). Antecedentes de la institucionalización de las organizaciones. Cuadernos de Gestión, 15(1), 15-38. https://www.redalyc.org/ pdf/2743/274339631001.pdf

Ejército NacionaldeColombia.(2011).FeenlaCausa.https://docplayer.es/62496294-Fe-en-la-causa-comportamiento-etico-superior.html

Ejército Nacional de Colombia. (2017a). Manual Fundamental de Liderazgo - MFE 6-22. Publicaciones Ejército.

Ejército Nacional de Colombia. (2017b). Manual Fundamental de Referencia Apoyo de la Defensa a la Autoridad Civil-MFRE 3-28. Publicaciones Ejército.

Ejército Nacional de Colombia. (2017c). Manual Fundamental Doctrina - MFE 1-01. Publicaciones Ejército. 
Ejército Nacional de Colombia. (2019). Manual de Campaña Acción Integral - MCE3-53.0. Publicaciones Ejército.

Ejército Nacional de Colombia. (s. f.). Departamento de Acción Integral y Desarrollo. https://www.ejercito.mil.co/conozcanos/organigrama/jefatura_estado_mayor_planeacion_253757/departamento_accion_integral_345389

Fernández-Santillán, J. F. (2018). Valor público, gobernanza y tercera vía. Convergencia: Revista de Ciencias Sociales, (78), 1405-1435. https://doi.org/10.29101/crcs.v25i78.10373

Fuerza Aérea Colombiana. (s. f.) Acción Integral. https://www.fac.mil.co/acci\%C3\%B3n-integral González, F. (2013). La hermenéutica y el método de las ciencias sociales. Cuadernos de Filosofía Latinoamericana, 34(109), 57-70.

Guiso, A. (1996). Métodos de investigación cualitativa. Universidad de Antioquia, 1-18. https:// aprendeenlinea.udea.edu.co/lms/moodle/file.php/563/Enfoques_de_Investigacion_ Cualitativa.pdf

Hayek, F. (1980). El ideal democrático y la contención del poder. Libertad y Leviatán. Revista del Centro de Estudios Públicos, (1), 12-75. https://www.cepchile.cl/cep/site/ docs/20160303/20160303183439/rev01_hayek1.pdf

Karan, C., \& Niño, A. (2018). Damasco: Evolución doctrinal para la consolidación de ganancias. Revista Experticia Militar, 5, 18-25. https://issuu.com/carlarami91/docs/revista-experticia-militar-5

López, O. (2017). Narcotráfico e insurgencia. Retos para el control territorial. En Memorias. Escuela Superior de Guerra. https://esdeguelibros.edu.co/index.php/editorial/catalog/ download $/ 18 / 15 / 34-1$ ? inline $=1$

Manterola, C., Astudillo, P., Arias, E., \& Claros. N. (2011). Revisiones sistemáticas de la literatura. Qué se debe saber acerca de ellas. https://www.researchgate.net/publication/251553765_Revisiones_sistematicas_de_la_literatura_Que_se_debe_saber_acerca_ de_ellas

Martínez, V. (2013). Paradigmas de investigación. Manual multimedia para el desarrollo de trabajos de investigación. Una visión desde la epistemología dialéctico crítica. https:// pics.unison.mx/wp-content/uploads/2013/10/7_Paradigmas_de_investigacion_2013.pdf

Ministerio de Defensa Nacional. (2019). Política de Defensa y Seguridad para la legalidad, el emprendimiento y la equidad. https:/www.mindefensa.gov.co/irj/go/km/docs/ Mindefensa/Documentos/descargas/Prensa/Documentos/politica_defensa_deguridad2019.pdf

Moliner, J. (2018). ¿Qué es la ética militar? http://www.ieee.es/Galerias/fichero/docs_ marco/2018/DIEEEM16-2018_EticaMilitar_JAMoliner.pdf

Montero, L. Sonja, Z., \& Newton, K. (2008). Confianza social, confianza política y satisfacción con la democracia. Revista Española de Investigaciones Sociológicas, (122), 11-54. http:// www.redalyc.org/pdf/997/99715236001.pdf

Montoya, M. (2007). La Acción Integral: Una estrategia para ganar la guerra. Centro de Estudios Estratégicos sobre Seguridad y Defensa Nacional. https://doi.org/10.25062/19008325.151 
Orjuela, L. (2000). La debilidad del Estado colombiano en tiempos del neoliberalismo y el conflicto armado. Colombia Internacional, (49-50), 104-116. https://revistas.uniandes. edu.co/doi/pdf/10.7440/colombiaint49-50.2000.05

Pérez, G. (2003). Investigación cualitativa. Retos e interrogantes. I. Métodos. La Muralla.

Serra, A. (2008). La gestión para resultados en las organizaciones públicas. En F. Longo \& I. Tamayo (eds.), Los escenarios de la gestión pública del siglo XXI (pp. 111-152). Bellaterra.

Registro Único de Víctimas (RUV). (2017). 9 de abril. \#Unidos por las víctimas [afiche]. http:// www.unidadvictimas.gov.co/sites/default/files/documentosbiblioteca/factsheet_9A2017.pdf

Rojas, J. (2019, marzo). Fe en Colombia: Aporte al compromiso bicentenario del Ejército Nacional para proteger a los colombianos. Revista Fuerzas Militares, 42(247), 11-20. https://issuu.com/esdeguecol/docs/247

UNESCO. (s. f.). Necesidades básicas. http://vocabularies.unesco.org/browser/thesaurus/es/ page/concept3614

Zartman, W. (1995). Collapsed States: The disintegration and restoration of legitimate authority. Lynne Rienner Publisher. 